\title{
QUANTIFICAÇÃO DE MATÉRIA ESTRANHA EM POLPAS CONGELADAS DE CAJÁ, CAJU E ACEROLA PRODUZIDAS E COMERCIALIZADAS NOS ESTADOS DO CEARÁ E RIO GRANDE DO NORTE
}

TEREZINHA FEITOSA *

MARIA DO SOCORRO ROCHA BASTOS *

MARIA ELISABETH BARROS OLIVEIRA *

CELLI RODRIGUES MUNIZ *

SÔNIA COELHO ABREU OLIVEIRA ***

\begin{abstract}
Como parte de um projeto que busca subsídios para o estabelecimento de Padrões de Identidade e Qualidade (P.I.Q) de polpa de frutas tropicais congeladas, este trabalho teve como objetivo avaliar amostras de polpas de acerola, cajá e caju produzidas e comercializadas nos Estados do Ceará e Rio Grande do Norte, quanto a presença de material estranho. Das 45 amostras submetidas a avaliação microscópica, $86,36 \%$ revelaram presença de material estranho, com predominância de fragmentos de insetos. Dentre os sabores analisados constatou-se que as amostras de acerola apresentaram maior quantidade de sujidades, seguidas pelas de cajá e caju. Como as amostras não atenderam as recomendações dos padrões estabelecidos para suco de frutas sugere-se 0 estabelecimento de práticas de controle sanitário para a produção de polpa de frutas.
\end{abstract}

\section{INTRODUÇÃO}

A polpa de frutas atende necessidades de vários segmentos da indústria de produtos alimentícios, tais como as de sucos naturais, sorvetes, laticínios, balas, doces, geleias, entre outras. No Brasil, a polpa industrializada destina-se principalmente à produção de sucos concentrados para o abastecimento do mercado interno e para exportação (7).

Uma participação mais efetiva do Brasil no mercado mundial de frutas vem ocorrendo, nos últimos anos, com perspectivas promissoras devido a uma série de fatores que indicam grandes vantagens competitivas do País no

* Pesquisadoras da EMBRAPA/Centro Nacional de Pesquisa de Agroindústria Tropical (CNPAT).

** Assistente de pesquisa da EMBRAPA/CNPAT.

*** Técnica do Núcleo de Tecnologia do Ceará (NUTEC). 
mercado externo (8). O perfil das exportações brasileiras de frutos, nos últimos 15 anos, apresenta tendência crescente para produto processado, principalmente na forma de polpa e suco. Menor exigência quanto à qualidade da matéria-prima, facilidades no transporte e conservação, a existência de agroindústria relativamente bem estruturada e a amplitude do mercado nacional são fatores que evidenciam grande potencial brasileiro para exportação de produtos processados de frutas. Todavia, sua atuação não tem sido satisfatória nestes mercados, sobretudo quando comparada com as de outros países exportadores, onde estão radicados grandes oligopólios internacionais, como Tailândia e Filipinas (5).

A comercialização de produtos industrializados, mesmo em menor quantidade é mais vantajosa em termos de receita gerada, que a do produto "in natura" (1). A vantagem dos processados em termos de preço final torna-se evidente segundo dados do Banco do Brasil/CEDEX (2).

O sucesso para se ingressar no mercado agroindustrial depende da capacidade ou habilidade de se competir com outras firmas já estabelecidas. Notadamente no setor de polpa de frutas, há que se considerar a existência de número expressivo de pequenas firmas que podem exercer pressão competitiva sobre o mercado, comercializando produtos com preços inferiores aos dos competidores tradicionais. Isto ocorre em função dos baixos custos operacionais deste seguimento, obtidos principalmente pela não observância das normas higiênicosanitárias e de qualidade das empresas mais conceituadas. Ademais, as microempresas deste setor geralmente operam à margem das instâncias federais, estaduais e municipais de arrecadação tributária (9).

A presença de materiais estranhos pode diminuir a aceitabilidade do produto sob o ponto de vista estético, uma vez que fabricantes, consumidores e órgãos de fiscalização esperam que os alimentos estejam inteiramente livres de material estranho. A matéria-prima pode ser contaminada ainda no campo ou durante o manuseio, processo tecnológico, armazenamento e distribuição inadequados (4).

Sob o ponto de vista da higiene é muito importante isolar matérias estranhas dos alimentos, como insetos e seus fragmentos, excrementos e pêlos de roedores, pois podem indicar o nível de contaminação e as práticas de higiene utilizadas na sua produção (3).

Este trabalho teve como objetivo avaliar a qualidade sanitária mediante detecção e identificação de contaminantes em polpa de cajá, caju e acerola produzidas e comercializadas nos Estados do Ceará e Rio Grande do Norte, pelo emprego de técnicas adequadas de isolamento para o exame microscópico. 
No período de janeiro à abril de 1996 foram examinadas 45 amostras de polpa de frutas sabor acerola, cajá e caju, em sua maioria adquiridas, diretamente dos produtores e pequena quantidade nos supermercados dos Estados do Ceará e Rio Grande do Norte.

As amostras foram coletadas em embalagens de $1 \mathrm{~kg}$, subdivididas em alíquotas de $100 \mathrm{~g}$ de acordo com a embalagem original de comercialização das empresas, transportadas para o laboratório em caixa de material isotérmico contendo gelo e acondicionadas em freezer. A análise e determinação de matérias estranhas presentes nos alimentos pode ser realizada pelo método de observação macroscópica ou por meio de métodos de isolamento para a detecção microscópica. Neste trabalho utilizou-se o método de flutuação, para identificação de sujidades leves, de acordo com as recomendações de KRAMER \& TWIGG (6).

Colocou-se $100 \mathrm{~g}$ da amostra em um frasco de Wildman juntamente com água destilada e óleo de rícino. Agitou-se durante 2 minutos e adicionou-se água a temperatura de $55-75^{\circ} \mathrm{C}$. Deixou-se o frasco em repouso por alguns minutos, agitando-o ocasionalmente. Raspou-se as bolhas de óleo da parede do frasco com o auxílio do bastão de agitação. Quando o óleo parou de subir e a fase aquosa ficou bem definida, adicionou-se água quente bem devagar, trazendo o óleo até o gargalo. Levantou-se o agitador vagarosamente até a camada superior do óleo e fechou-se a abertura do frasco. Transferiu-se a fase oleosa para becker. Repetiu-se a decantação do óleo no becker várias vezes para total remoção do mesmo. Descartouse o conteúdo do frasco e completou-se cerca de $3 / 4$ da capacidade do frasco com água quente. Transferiu-se o óleo coletado mais as águas de lavagem para um frasco e separou-se o óleo, como anteriormente indicado num becker. Filtrou-se sob vácuo a amostra coletada no becker, removeuse o papel de filtro e observou-se através de estereoscópio. Contou-se e identificou-se o material observado.

\section{RESULTADOS E DISCUSSÃO}

Examinando os resultados referentes às polpas de cajá (Tabela 1) constata-se que, das 14 amostras analisadas 85,7\% apresentaram fragmentos de insetos (com variação de 01 a 50 fragmentos/100 g), 7,1\% apresentaram insetos inteiros, $7,1 \%$ apresentaram larvas de inseto e $42,8 \%$ apresentaram outras matérias estranhas (pêlos, escrementos de insetos, grãos de areia), com variação de 02 a 18 sujidades $/ 100$ g da amostra. 
TABELA 1 - AVALIAÇÃO DA PRESENÇA DE SUJIDADES EM POLPAS DE FRUTAS COMERCIALIZADAS NOS ESTADOS DO CEARÁ E DO RIO GRANDE DO NORTE

\begin{tabular}{|c|c|c|c|c|c|c|c|c|c|}
\hline $\begin{array}{c}\text { POLPA } \\
\text { DE CAJÁ }\end{array}$ & $\begin{array}{l}\text { FRAGMENTOS } \\
\text { DE INSETOS } \\
\left(\mathrm{N}^{\circ} / 100 \mathrm{~g}\right)\end{array}$ & $\begin{array}{l}\text { INSETOS } \\
\text { INTEIROS }\end{array}$ & $\begin{array}{c}\text { OUTRAS } \\
\text { SUJIDADES }\end{array}$ & $\begin{array}{l}\text { LARVAS } \\
(\mathrm{N} / 100 \mathrm{~g})\end{array}$ & $\begin{array}{c}\text { POLPA } \\
\text { DE CAJÁ }\end{array}$ & $\begin{array}{l}\text { FRAGMENTOS DE } \\
\text { INSETOS (No/100 g) }\end{array}$ & $\begin{array}{l}\text { INSETOS } \\
\text { INTEIROS }\end{array}$ & $\begin{array}{c}\text { OUTRAS } \\
\text { SUJIDADES }\end{array}$ & $\begin{array}{l}\text { LARVAS } \\
\text { (N\%/100 g) }\end{array}$ \\
\hline AMOSTRA & \multicolumn{4}{|c|}{ CEARÁ } & AMOSTRA & \multicolumn{4}{|c|}{ RIO GRANDE DO NORTE } \\
\hline 1 & 00 & - & - & - & 1 & 02 & - & - & - \\
\hline 2 & 07 & - & - & - & 2 & 12 & - & 18 & - \\
\hline 3 & 00 & - & - & - & 3 & 01 & - & 03 & - \\
\hline 4 & 06 & - & - & - & - & - & - & - & - \\
\hline 5 & 31 & - & 17 & - & - & - & - & - & - \\
\hline 6 & 03 & - & 03 & - & 6 & 06 & 01 & 10 & - \\
\hline 7 & 10 & - & 03 & 01 & - & - & - & - & - \\
\hline 8 & 15 & - & 02 & - & 8 & 50 & - & - & - \\
\hline 9 & 17 & - & 08 & - & - & - & - & - & - \\
\hline \multirow{2}{*}{\multicolumn{3}{|c|}{ ESTADO }} & \multicolumn{7}{|c|}{ FRAGMENTOS DE INSETOS (\%) } \\
\hline & & & \multicolumn{4}{|c|}{ PRESENÇA } & \multicolumn{3}{|c|}{ AUSÊNCIA } \\
\hline \multicolumn{3}{|c|}{ CEARÁ } & \multicolumn{4}{|c|}{77,77} & \multicolumn{2}{|c|}{22,22} & \\
\hline \multicolumn{3}{|c|}{ RIO GRANDE DO NORTE } & \multicolumn{3}{|c|}{100,00} & & \multicolumn{2}{|c|}{0,00} & \\
\hline \multicolumn{3}{|c|}{ TOTAL } & \multicolumn{3}{|c|}{85,71} & & \multicolumn{2}{|c|}{14,28} & \\
\hline
\end{tabular}


TABELA 2 - AVALIAÇÃO DA PRESENCCA DE SUJIDADES EM POLPAS DE FRUTAS COMERCIALIZADAS NOS ESTADOS DO CEARẢ E DO RIO GRANDE DO NORTE

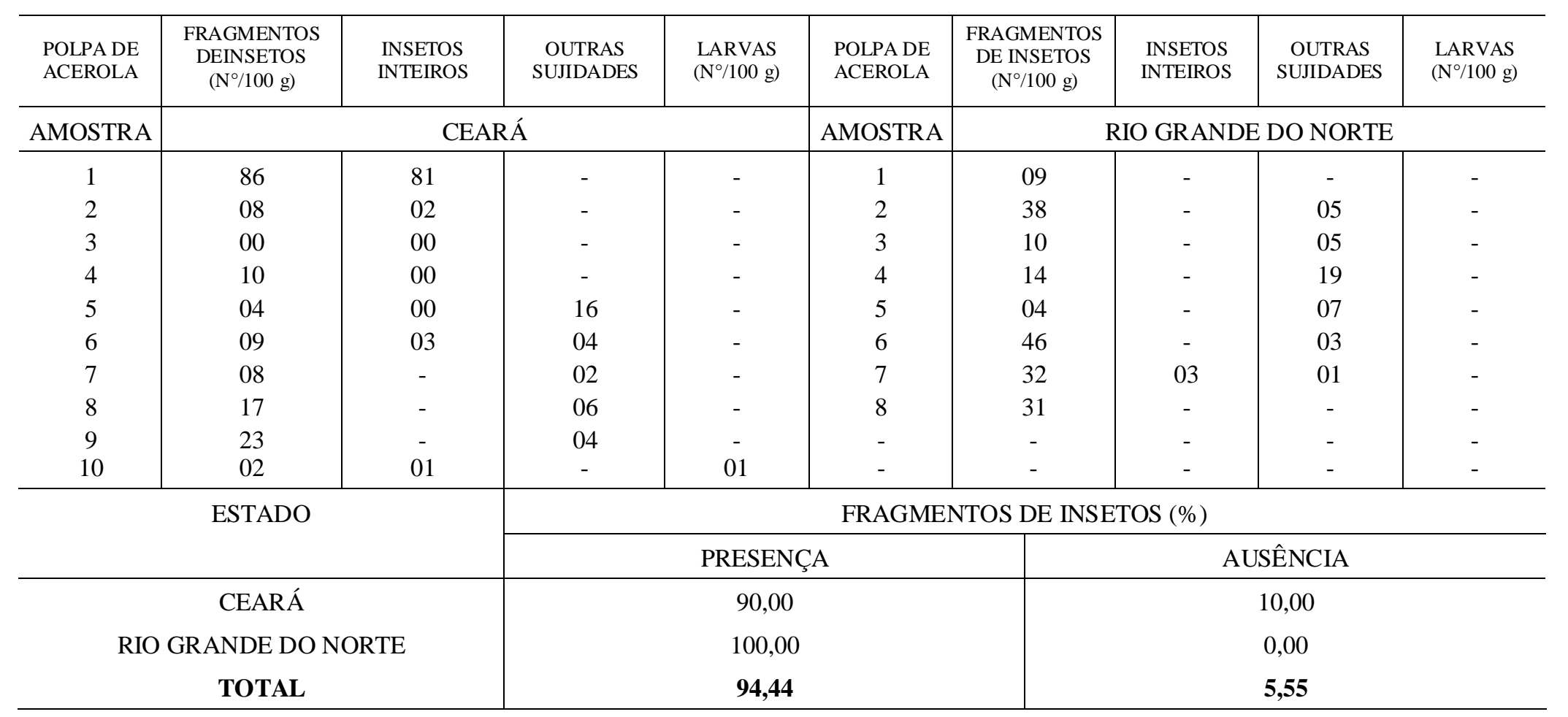


TABELA 3 - AVALIAÇÃO DA PRESENÇA DE SUJIDADES EM POLPAS DE FRUTAS COMERCIALIZADAS NOS ESTADOS DO CEARÁ E DO RIO GRANDE DO NORTE

\begin{tabular}{|c|c|c|c|c|c|c|c|c|c|}
\hline $\begin{array}{c}\text { POLPA } \\
\text { DE CAJU }\end{array}$ & $\begin{array}{l}\text { FRAGMENTOS DE } \\
\text { INSETOS } \\
\left(\mathrm{N}^{\circ} / 100 \mathrm{~g}\right)\end{array}$ & $\begin{array}{l}\text { INSETOS } \\
\text { INTEIROS }\end{array}$ & $\begin{array}{l}\text { OUTRAS } \\
\text { SUJIDADES }\end{array}$ & $\begin{array}{l}\text { LARVAS } \\
\left(\mathrm{N}^{\circ} / 100 \mathrm{~g}\right)\end{array}$ & $\begin{array}{l}\text { POLPA DE } \\
\text { CAJU }\end{array}$ & $\begin{array}{l}\text { FRAGMENTOS } \\
\text { DE INSETOS } \\
\text { (No/100 g) }\end{array}$ & $\begin{array}{l}\text { INSETOS } \\
\text { INTEIROS }\end{array}$ & $\begin{array}{c}\text { OUTRAS } \\
\text { SUJIDADES }\end{array}$ & $\begin{array}{l}\text { LARVAS } \\
(\mathrm{N} / 100 \mathrm{~g})\end{array}$ \\
\hline AMOSTRA & \multicolumn{3}{|c|}{ CEARÁ } & & AMOSTRA & \multicolumn{4}{|c|}{ RIO GRANDE DO NORTE } \\
\hline $\begin{array}{c}1 \\
2 \\
3 \\
4 \\
5 \\
6 \\
7 \\
8 \\
9 \\
10\end{array}$ & $\begin{array}{l}00 \\
10 \\
00 \\
22 \\
- \\
- \\
20 \\
38 \\
24 \\
09\end{array}$ & & $\begin{array}{c}- \\
- \\
02 \\
19 \\
09\end{array}$ & - & $\begin{array}{l}1 \\
2\end{array}$ & $\begin{array}{l}03 \\
34 \\
\\
- \\
- \\
10\end{array}$ & - & $\begin{array}{l}49 \\
- \\
-\end{array}$ & - \\
\hline \multirow{2}{*}{\multicolumn{3}{|c|}{ ESTADO }} & \multicolumn{7}{|c|}{ FRAGMENTOS DE INSETOS (\%) } \\
\hline & & & \multicolumn{4}{|c|}{ PRESENÇA } & \multicolumn{3}{|c|}{ AUSÊNCIA } \\
\hline \multicolumn{3}{|c|}{ CEARÁ } & \multicolumn{4}{|c|}{75,00} & \multicolumn{3}{|c|}{25,00} \\
\hline \multicolumn{3}{|c|}{ RIO GRANDE DO NORTE } & \multicolumn{4}{|c|}{100,00} & \multicolumn{3}{|c|}{0,00} \\
\hline \multicolumn{3}{|c|}{ TOTAL } & \multicolumn{4}{|c|}{81,81} & \multicolumn{3}{|c|}{18,18} \\
\hline
\end{tabular}

(-) Amostras não analisadas devido a não existência de polpas no comércio local na data da coleta. 
Os resultados obtidos para as polpas de acerola (Tabela 2) evidenciaram que, de 18 amostras analisadas, 94,4\% apresentaram fragmentos de insetos (com variação de 02 a 86 fragmentos/100 g), 27,7\% apresentaram insetos inteiros (com variação de 01 a 81 insetos/100 g), 5,5\% apresentaram larvas de inseto e $61,1 \%$ apresentaram outras sujidades, que variaram de 01 a 19 sujidades/100 g da amostra.

Das 11 amostras de polpas de caju (Tabela 3), 81,8\% apresentaram fragmentos de insetos (com variação de 03 a 38 fragmentos/100 g), 9,0\% apresentaram larvas de insetos e $36,3 \%$ apresentaram outras sujidades, que variaram de 02 a 49 sujidades/100 g da amostra.

\title{
4 CONCLUSÃO
}

De acordo com os resultados do presente estudo pode-se concluir que:

- Entre os sabores analisados, a acerola foi o que apresentou maior quantidade de material estranho e o cajá a menor. O caju apresentou uma quantidade intermediária de sujidades entre os dois outros acima citados.

- Do total de amostras de polpa analisadas (45), apenas 03 (uma de cada sabor) apresentaram qualidade satisfatória.

- Tais resultados evidenciam a necessidade de ação fiscalizadora mais intensa no que diz respeito a polpa de frutas, bem como a elaboração de um Padrão de Identidade e Qualidade do referido produto.

\begin{abstract}
As a part of the project with the aim to define Quality and Identity Standards (QIS) for tropical fruit frozen pulps, this work has been carried out with the objective of dirtiness evaluation in samples of acerola (Malpighia punicifolia), yellow mombin (cajá) (Spondias mombin L), and cashew (caju) (Anacardium occidentale) fruits pulps produced and commercialized within the States of Ceará and Rio Grande do Norte (Brazil). Samples in number of 45, were submitted to microscopic evaluation, adapting the AOAC's methods of flutation for light dirtiness. The results showed up that $86.36 \%$ of the samples contained dirtiness, mainly being scraps of insects. Among these analysed fruit pulps, the samples of acerola occurred to carry the major dirtiness number, followed by yellow mombin and cashew fruit pulp samples. The results of the analyses indicated that no one of the samples filled out the established quality standards for tropical fruit juices. Practices of sanitary control must be defined and adapted for the production of fruit pulps.
\end{abstract}




\section{REFERÊNCIAS BIBLIOGRÁFICAS}

1 ALMEIDA, L. A. S. B., BLEINROTH, E. W., MORETTI, V.A., BICUTO NETO, L. C., RIVELLI, P.B. Comercialização de frutas tropicais. Campinas : ITAL, 1987. 106 p. (Estudos Econômicos, 25).

2 BANCO DO BRASIL/CEDEX. Departamento de Comércio Exterior. Banco de dados. Rio de Janeiro, 1993.

3 BARBIERI, M. K., SERRANO, A. M. Princípios gerais para isolamento e identificação de matérias estranhas em alimentos. Coletânea ITAL, Campinas, v. 25, n. 2, p. 123-132, jul/dez. 1995.

4 BARBIERI, M. K. Microscopia em alimentos: identificação histológica, isolamento e detecção de material estranho em alimentos: manual técnico. Campinas : ITAL, 1990.

5 INTERNATIONAL fruits world. Switzerland : Agro Press, 1993/1994.

6 KRAMER, A., TWIGG, B. Quality control for the food industry. 3.ed. Westport: AVI, 1970. v. 1, p. 155-172.

7 BRASIL. Produção de polpa de fruta tratada termicamente e congelada. Brasília, 1995. (Série Perfis Agroindustriais).

8 SAVITCI, L. A., GASPARINO FILHO, J., MORETTI, V. A. Perfil industrial e de mercado para suco de abacaxi. Coletânea ITAL, V. 25, n. 2, p. 153-168, jul./dez. 1995.

9 VILAS, A. T. Análise dos principais mercados internacionais de sucos e polpas de frutas tropicais. Brasília : EMBRAPA, 1992. 36 p. (Relatório não publicado). 